
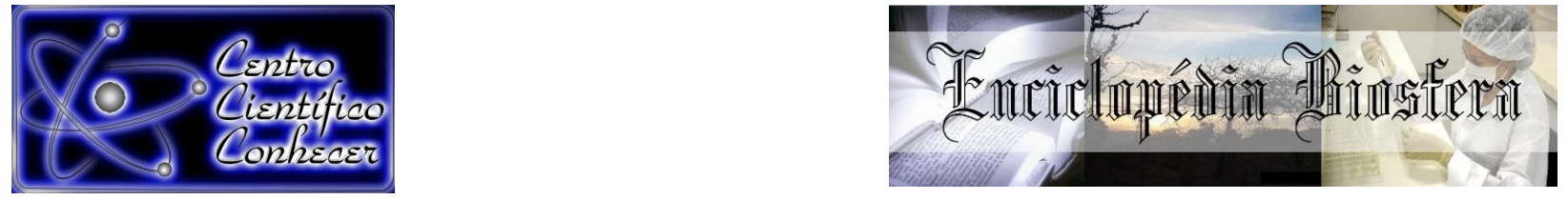

\title{
COMPARAÇÃO DA ANSIEDADE E DA CAPACIDADE COGNITIVA ENTRE IDOSOS PRATICANTES DE CAMINHADA E MUSCULAÇÃO
}

Rafael Hideki Abiko1; Silvia Harumi Tamura; Paulo Henrique Borges; Leonardo Pestillo de Oliveira ${ }^{4}$ S Sonia Maria Marques Gomes Bertolini ${ }^{5}$

${ }^{1}$ Mestrando em Promoção da Saúde, Centro Universitário de Maringá - UNICESUMAR, Maringá, Paraná, Brasil. Bolsista Capes. rafaelabiko@gmail.com

${ }^{2}$ Graduada em Fisioterapia, Centro Universitário de Maringá - UNICESUMAR, Assaí, Paraná, Brasil.

${ }^{3}$ Doutorando em Educação Física, Universidade Estadual de Maringá- UEM. Maringá, Paraná, Brasil.

${ }^{4}$ Doutor em Psicologia Social, Centro Universitário de Maringá - UNICESUMAR, Maringá, Paraná, Brasil. Bolsista do Instituto Cesumar de Ciência, Tecnologia e Inovação - ICETI.

${ }^{5}$ Doutora em Ciências Morfofuncionais, Centro Universitário de Maringá - UNICESUMAR, Maringá, Paraná, Brasil. Pesquisadora do Instituto Cesumar de Ciência, Tecnologia e Inovação - ICETI. sonia.bertolini@unicesumar.edu.br

Recebido em: 06/04/2018 - Aprovado em: 10/06/2018 - Publicado em: 20/06/2018 DOI: 10.18677/EnciBio_2018A97

Uma vida ativa é capaz de manter a autonomia funcional, retardando ou minimizando as alterações decorrentes do processo de envelhecimento. Neste contesto, o exercício físico atua de forma positiva para a saúde do idoso. O estudo objetivou comparar os níveis de ansiedade e capacidade cognitiva entre idosos praticantes de musculação e caminhada, bem como, verificar a correlação entre a ansiedade e o risco de quedas. A amostra foi constituída por 96 idosos de ambos os sexos, sendo 47 praticantes de caminhada e 49 praticantes de musculação. Para avaliação da ansiedade estado foi utilizado o Inventário de Ansiedade Traço-Estado e para avaliar a capacidade cognitiva o Mini Exame do Estado Mental (MEEM). Os resultados revelaram associação significativa entre o nível de escolaridade e o tipo de exercício físico $(p=0,03)$, correlação estatisticamente significativa do MEEM com a idade $(p=0,03)$ e com o nível de escolaridade $(p<0,01)$. Não foram verificadas diferenças significativas nos níveis de ansiedade e capacidade cognitiva entre os idosos praticantes de musculação e caminhada, sendo assim conclui-se que ambas as atividades podem contribuir para o envelhecimento ativo.

PALAVRAS-CHAVE: Exercício físico; Promoção da saúde; Saúde da terceira idade.

\section{COMPARISON THE STATE OF ANXIETY AND COGNITIVE CAPACITY BETWEEN ELDERLY PRACTICING WALKING AND STRENGTH TRAINING}

\begin{abstract}
An active life is able to maintain the functional autonomy, delaying or minimizing the changes due to the aging process, thus, the physical exercise acts in a positive way for the health of the elderly. The aim of this study was to compare the state of anxiety levels and the cognitive ability in elderly people who practice strength training and go walking, as well as, to verify the correlation between anxiety and the risk of falls.. The sample was represented of 96 elderly people of both genders. To evaluation the
\end{abstract}


state anxiety was used the Trait- State Anxiety Inventory (STAI- E) and the cognitive ability was used the Mini Mental State Examination (MMSE). The results showed a significant association among the level of schooling and the kind of exercise $(p=$ $0.03)$, a statistically significant correlation between MMSE and age $(p=0.03)$ and the level of schooling $(p<0.01)$. It can be concluded that there weren't identified significant differences in the levels of anxiety and cognitive ability among the elderly the go strength training.

KEYWORDS: Physical exercise; Health promotion; Health of the elderly.

\section{INTRODUÇÃO}

O envelhecimento ativo é definido como o processo de otimizar as oportunidades de saúde, participação e segurança, com o objetivo de melhorar a qualidade de vida à medida que as pessoas envelhecem. As abordagens do envelhecimento ativo podem desempenhar um papel importante na promoção da saúde mental de idosos (GALLI et al., 2016), uma vez que a ansiedade é altamente prevalente entre os idosos (MACHADO et al, 2016).

Entre os benefícios da atividade física para a saúde física e mental dos idosos, destacam-se a melhora do equilíbrio, da flexibilidade, da mobilidade, da coordenação motora, da independência funcional, da aptidão cardiorrespiratória, da auto estima, do bem-estar, da qualidade do sono, além diminuir a ansiedade (HELRIGLE et al., 2013). Sendo assim, os idosos praticantes de exercícios físicos são mais propensos a ter um envelhecimento saudável.

A caminhada é uma das atividades físicas mais praticadas entre os adultos. Por ser uma atividade de baixo custo e de fácil acesso, produz efeitos significativos relacionados à melhora da aptidão física e redução de risco de doenças cardiovasculares (QUEIROZ et al., 2013). Estudos apresentam a caminhada na melhora da ansiedade e depressão em pacientes na reabilitação de câncer de pulmão (CHEN et al., 2015), assim como, em pacientes com transtorno do pânico (LATTARI et al., 2015).

A importância da prática da musculação é evidente na vida do idoso, permitindo melhor desempenho físico, tornando-os menos suscetíveis às fraturas ósseas que geralmente acompanham os indivíduos da terceira idade (COELHO et al., 2010).

Os quadros de ansiedade são frequentes na população idosa (MACHADO, 2016). O exercício físico pode ser considerado uma alternativa não medicamentosa para o tratamento de distúrbios psicológicos como a ansiedade e alterações de humor, impactando positivamente na qualidade de vida do idoso (ANTUNES et al., 2005).

O comprometimento cognitivo dos idosos é associado com a ocorrência de quedas, sendo agravante para a capacidade funcional e qualidade de vida do idoso (CRUZ et al., 2015). Foi verificado que quanto mais grave é a função cognitiva, maior é o comprometimento da funcionalidade e do equilíbrio, ocorrendo um aumento no risco de quedas quando comparado com demências mais leves (BORTOLI et al., 2015). A função cognitiva também está correlacionada com a aprendizagem de execução de novas habilidades motoras, auxiliando nas avaliações das condições musculoesqueléticas do paciente, mas também a parte cognitiva (ALTERMANN et al., 2014). A importância da participação dos idosos em algum tipo de exercício físico contribui significativamente para a manutenção de um envelhecimento ativo, capaz de prevenir o declínio cognitivo e promover saúde ao idoso (VARGAS et al., 2014). 
Em relação as diversas modalidades de atividades físicas praticadas entre os idosos, de acordo com o American College of Sports Medicine, a caminhada é a mais comum e a musculação é a mais recomendada para retardar a sarcopenia (GOBBl et al, 2005).

Considerando os índices expressivos de ansiedade entre os idosos, e as considerações American College of Sports sobre os tipos de atividades físicas realizadas pela população idosa, este estudo teve como objetivo comparar os níveis de ansiedade e capacidade cognitiva entre idosos praticantes de musculação e caminhada, bem como, verificar a correlação entre a ansiedade e o risco de queda.

\section{MATERIAL E MÉTODOS}

Trata-se de um estudo transversal com abordagem quantitativa. A amostra foi constituída por 96 idosos com idade acima de 60 anos (47 praticantes caminhada e 49 praticantes de musculação) residentes na cidade de Maringá-PR. A amostra foi selecionada por conveniência em 10 academias da região central da cidade, e nos principais pontos de caminhada, como o Parque do Ingá e Bosque II. Como critério de inclusão, foram selecionados os idosos que praticavam a musculação ou a caminhada por mais de 6 meses ininterruptos e com frequência semanal de pelo menos 150 minutos no exercício físico. Foram excluídos os idosos que apresentavam alguma deficiência física ou psíquica e patologias que comprometessem os resultados da pesquisa.

Para avaliar o estado de ansiedade foi utilizado a primeira parte do Inventário de Ansiedade Traço-Estado (IDATE-E), proposto por Spielbergeret al. (1970), traduzido e adaptado para o Brasil (BIAGGIO et al, 1979). O instrumento é uma escala de autorrelato que depende da reflexão consciente do sujeito no processo da avaliação do seu estado de ansiedade, assim como de características de sua personalidade. É caracterizado por sentimentos desagradáveis de tensão e apreensão, percebidos conscientemente, sendo que eles podem variar em intensidade, de acordo com o perigo percebido pela pessoa e a alteração no tempo. Este instrumento apresenta 20 afirmações para as quais os voluntários indicam a intensidade naquele momento por meio de uma escala likert de quatro pontos (1 a 4). O escore total de cada escala varia de 20 a 80 , sendo que os valores mais altos indicam maiores níveis de ansiedade. A fim de evitar influência da "tendência à aquiescência" nas respostas, alguns itens são pontuados de maneira inversa, isto é, as respostas marcadas com 1,2, 3 ou 4 recebem o valor de 4, 3, 2 ou 1 respectivamente. $\mathrm{Na}$ escala IDATE-E, existem dez itens computados desta maneira $(1,2,5,8,10,11,15,16,19$ e 20$)$.

No que se refere a capacidade cognitiva foi utilizado o Mini exame do estado mental (MEEM), este instrumento é fundamental para o rastreio do comportamento cognitivo (BRUCKI et al., 2003). No Brasil o MEEM foi traduzido e validado por Bertolucci et al. (1994). O MEEM é constituído por 5 categorias que reúnem diferentes questões, das quais pode atingir um total máximo de 30 pontos. As questões têm como finalidade avaliar diversos campos cognitivos: orientação para tempo (5 pontos), orientação para local (5 pontos), registro de 3 palavras (3 pontos), atenção e cálculo (5 pontos), lembrança das 3 palavras (3 pontos), linguagem (8 pontos), e capacidade construtiva visual (1 ponto).

Utilizou-se ainda um questionário com questões referentes ao grau de escolaridade,e a ocorrência de queda, elaborado pelos próprios pesquisadores do estudo. 
A presente pesquisa foi aprovada pelo Comitê de ética em pesquisa com seres humanos do Centro Universitário de Maringá (Parecer $\left.n^{\circ} 1.805 .099\right)$. Todos os participantes assinaram o termo de consentimento livre e esclarecido e responderam as perguntas do questionário de modo individual com os avaliadores realizando as perguntas oralmente, ocorrendo sempre ao início ou ao final de seu respectivo exercício físico no próprio estabelecimento de sua prática.

Para análise dos dados utilizou-se o teste Kolmogorov-Smirnov para verificar a normalidade dos dados; o teste $t$ independente para comparar os resultados do MEEM e do IDATE- E entre os grupos; o teste Qui-quadrado pelo método linha por linha para verificar associações existentes entre o tipo de exercício físico (caminhada ou musculação) com os níveis de escolaridade dos idosos. Para verificar a correlação entre variáveis numéricas/categóricas do estudo, utilizou-se a correlação de Spearman. Os dados foram analisados no programa estatístico Statistical Package for the Social Sciences (SPSS) 20.0. O nível de significância adotado foi de $5 \%(p \leq 0,05)$.

\section{RESULTADOS E DISCUSSÃO}

Ao comparar as categorias do MEEM com os grupos de idosos, verificou-se diferença estatisticamente significativa na categoria evocação, no qual aqueles que praticavam musculação apresentavam melhores escores de memória (Tabela 1). De acordo com Trewartha et al. (2014), a função cognitiva está relacionada com a aprendizagem de novas habilidades motoras. $O$ fato dos idosos praticantes de musculação realizarem diferentes exercícios quando comparado à caminhada que é sempre o mesmo gesto motor, pode ser a explicação para melhor pontuação na evocação encontrada no referido grupo. Outra explicação para este resultado é que os sujeitos que praticavam a musculação eram os que tinham maiores níveis de escolaridade, consequentemente estavam mais habituados a utilizarem a memória para gravarem os conteúdos mais complexos, sugerindo que as atividades que estimulam a mente e o corpo dos idosos ativos, proporcionam mais autonomia e qualidade de vida (MELLO et al., 2012).

Tabela 1. Comparação da idade e das categorias do MEEM dos idosos praticantes de caminhada e musculação.

\begin{tabular}{cccccc}
\hline \multirow{2}{*}{ Variáveis } & $\begin{array}{c}\text { Caminhada } \\
(\mathbf{n}=\mathbf{4 7})\end{array}$ & $\begin{array}{c}\text { Musculação } \\
(\mathbf{n}=\mathbf{4 9})\end{array}$ & \multirow{2}{*}{$\mathbf{t}$} & $\mathbf{p}$ \\
\cline { 2 - 3 } & $\mathbf{M}$ & $\mathbf{M}$ Média $\pm \mathbf{d p}$ & $\mathbf{M} \pm \mathbf{d p}$ & & \\
\hline Idade (anos) & $68,34 \pm 6,85$ & $67,73 \pm 7,68$ & 0,40 & 0,68 \\
Orientação (pont.) & $9,74 \pm 0,48$ & $9,85 \pm 0,35$ & $-1,28$ & 0,20 \\
Memória Imediata (pont.) & $2,93 \pm 0,32$ & $2,93 \pm 0,24$ & $-0,04$ & 0,96 \\
Atenção e cálculo (pont.) & $2,53 \pm 1,85$ & $3,06 \pm 1,88$ & $-1,38$ & 0,16 \\
Evocação (pont.) & $2,00 \pm 0,90$ & $2,34 \pm 0,80$ & $-1,98$ & $0,05 *$ \\
Linguagem (pont.) & $8,21 \pm 0,99$ & $8,10 \pm 1,08$ & 0,79 & 0,60 \\
\hline
\end{tabular}

Nota: pont.=pontuação no teste; $p \leq 0,05$.

$\mathrm{Na}$ tabela 2, verifica-se que houve uma associação significativa entre o nível de escolaridade e o tipo de exercício físico que o idoso realiza. 
Tabela 2. Associação entre a escolaridade e o tipo de exercício físico praticado por idosos.

\begin{tabular}{lcccc}
\hline & Caminhada & Musculação & $\mathbf{X}^{\mathbf{2}}$ & $\mathbf{p}$ \\
\hline Ensino fundamental I (4 $\mathbf{4}^{\circ}$ série completa) & $8(8,3 \%)$ & $4(4,2 \%)$ & & \\
$\begin{array}{l}\text { Ensino fundamental II ( } \mathbf{8}^{\circ} \text { série } \\
\text { completa) }\end{array}$ & $10(10,4 \%)$ & $8(8,3 \%)$ & & \\
Ensino médio & $13(13,5 \%)$ & $9(9,4 \%)$ & & \\
Ensino superior & $16(16,7 \%)$ & $28(29,2 \%)$ & & \\
\hline
\end{tabular}

Neste estudo as pessoas com maior escolaridade faziam musculação, o que está de acordo com outro estudo em que idosos com maior escolaridade eram praticantes de grupos de hidroginástica e musculação (OLIVEIRA et al., 2014). O fato da musculação ser um tipo de atividade física programada exigindo mais das funções psíquicas superiores pela sua maior complexidade que a caminhada, evolvendo intensidade, frequência entre outras variáveis, poderia justificar essa associação.

Independente da modalidade de exercício físico praticado pelos idosos, verificou-se ainda, que aqueles que apresentaram maiores valores de MEEM eram os que possuíam maior nível de escolaridade $(r=0,38 ; p<0,01)$, e também eram os idosos mais novos $(r=-0,21 ; p=0,03)$. Embora o estudo tenha encontrado correlações estatisticamente significativas, estas foram consideradas correlações fraca e moderada.

$\mathrm{Na}$ literatura tem sido apontadas associações significativas entre idosos que têm déficit cognitivo ou que apresentam menores valores do MEEM, com idosos que têm uma menor força de preensão palmar (PADUBIDRI et al., 2014), indicando que o trabalho na musculação pode contribuir para o aumento da força muscular, e consequentemente, ajudar na melhora da função cognitiva.

Outras modalidades de exercícios físicos também têm sido estudadas como a prática de caratê, que demonstrou melhores resultados no desempenho cognitivo dos idosos, comparado aos que praticavam caminhada não supervisionada e aos que não praticavam nenhuma atividade física regular (DASCAL et al., 2013).

Conforme mostrado na figura 1, ao comparar os dados do MEEM e do IDATE estado entre os grupos, não foi identificado diferença significativa $(p=0,14$ e $p=$ 0,68 ), indicando que ambos os exercícios contribuem para os idosos terem bons índices de capacidade cognitiva e um baixo nível de ansiedade estado. Uma explicação para estes resultados é que ambos os exercícios físicos proporcionam um aumento do fluxo sanguíneo cerebral, ofertando um melhor aporte de nutrientes assim como, o aumento do número de neurotransmissores melhorando a capacidade cognitiva (RAMOS; CHAGAS, 2015). A longo prazo, o exercício físico é capaz de promover adaptações cerebrais e plasticidade cerebral que melhora o desempenho cognitivo (FILHO et al., 2014). 


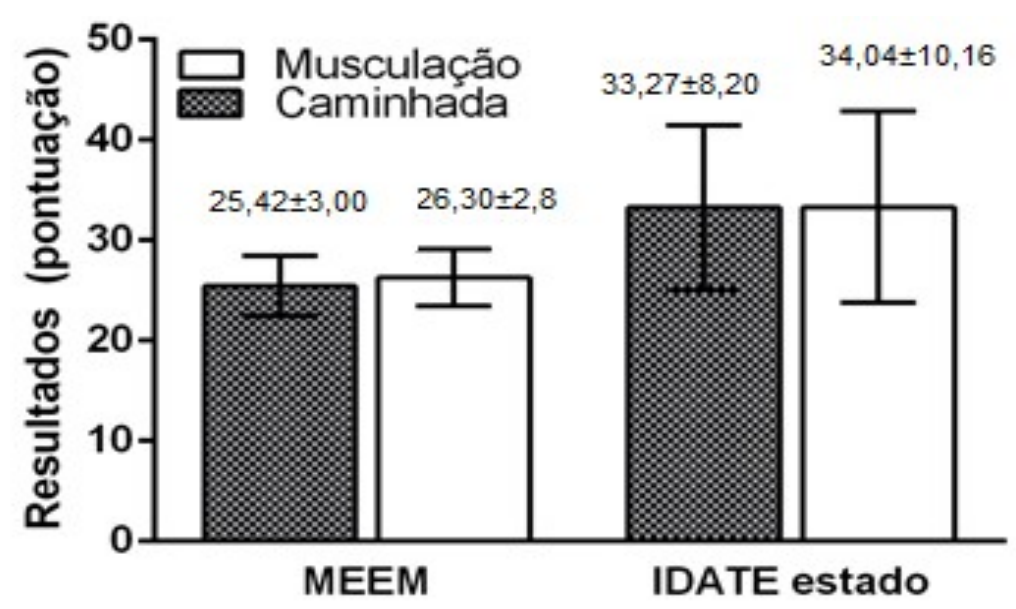

Figura 1. Comparação do nível de ansiedade estado e da capacidade cognitiva entre idosos praticantes de musculação e caminhada.

O exercício aeróbio pode melhorar a ansiedade (ARAÚJO et al., 2007). Os exercícios aeróbios de intensidade até o limiar de lactato demonstraram ser apropriados para o tratamento de pessoas com ansiedade patológica.

Os idosos praticantes de musculação apresentam maior força muscular dinâmica e isométrica em membros superiores e inferiores quando comparados a sedentários ou praticantes de outro exercício físico (COELHO et al., 2014). Contribui também para prevenção de doenças como a sarcopenia. Araújo et al. (2017) realizaram um estudo com mulheres com uma média de idade acima dos cinquenta anos que participaram de um treinamento resistido com pesos, 3 vezes por semana, com duração de 20 a 30 minutos, e verificaram menores pontuações nos escores de ansiedade e depressão no grupo teste, quando comparado ao controle sedentário.

Uma outra correlação significativa encontrada no estudo foi do IDATE com as quedas $(p<0,05)$, ou seja os idosos com maiores níveis de ansiedade, eram os que possuíam menor risco de queda. No entanto, a média dos dois grupos de exercício físico apresentou níveis baixos de ansiedade.

Estudos que avaliaram o risco de quedas em idosos revelam que uma possível explicação para a baixa incidência de quedas na população idosa ativa ocorre em virtude de serem mais saudáveis ou por apresentarem doenças mais controladas e por serem mais independentes (BRETAN et al., 2010), outro fator que nosso estudo não controlou, sendo considerado outra limitação. Contudo, o estudo torna-se relevante para que novas ações e políticas públicas possam ser implementadas com o intuito de promover mais saúde física e mental à população de idosos com aumento expressivo nos últimos anos.

Não foram encontradas diferenças estatísticas referentes a idade (Tabela 1) e o volume de treino semanal entre os grupos pesquisados (Figura 2). 


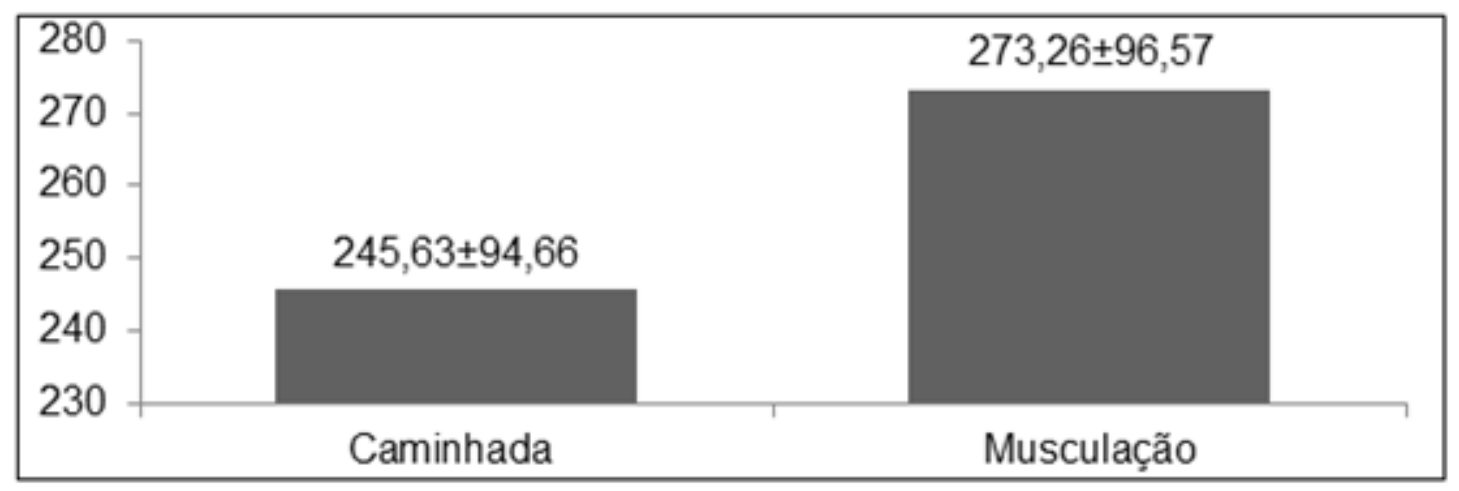

Figura 2. Comparação do volume de treino entre os idosos praticantes de caminhada e musculação. Nota: os valores se referem ao total de minutos da prática de exercício físico realizada por semana $(p=0,16)$.

No presente estudo, a ausência de significância entre as idades e o volume de treino dos dois grupos estudados, minimizam interferências nos resultados devido à homogeneidade dos grupos.

No estudo de Domiciano et al. (2014), a idade juntamente com o nível de escolaridade teve forte influência na pontuação total do MEEM. O mesmo ocorreu com o volume de treino abordado no estudo com mulheres pós-menopausa divididas em grupo pouco ativas (GPA: realizavam menos que 150 minutos por semana) e o grupo ativas (GA: realizavam mais que 150 minutos por semana); no qual o grupo GA apresentou melhor desempenho cognitivo para orientação temporal; e o escore geral do MEEM também foi maior, embora a diferença não tenha sido significativa (RAMOS; CHAGAS, 2015).

Todo estudo realizado por meio questionário tem questões subjetivas que capazes de levar à perda de precisão e confiabilidade, o que pode ser considerado uma limitação da pesquisa. Outro fato é que se trata de um estudo com delineamento transversal, não permitindo verificar a relação causal entre as variáveis.

\section{CONCLUSÃO}

Conclui-se que não existem diferenças significativas nos níveis de ansiedade e capacidade cognitiva entre os idosos praticantes de musculação e caminhada, sendo assim, infere-se que ambas as atividades podem contribuir para o envelhecimento ativo.

Acredita-se que os escores semelhantes encontrados com a utilização do Mini exame do estado mental e do Inventário e ansiedade estado, nos grupos avaliados podem contribuem para escolha do idoso por qualquer uma das modalidades de exercício físico (caminhada ou musculação), quando os efeitos desejáveis se relacionam a melhora nas variáveis ansiedade ou capacidade cognitiva.

\section{REFERÊNCIAS}

ALTERMANN, C.D.C.; MARTINS, A. S.; CARPES, F. P.; MELLO-CARPES, P. B. Influence of mental practice and movement observation on motor memory, cognitive function and motor performance in the elderly. Brazilian Journal of Physical Therapy. v.18, n.2, p.201- 209, 2014. Disponível em: http://www.scielo.br/pdf/rbfis/v18n2/pt_1413-3555-rbfis-18-02-00201.pdf. doi: 10.1590/S1413-35552012005000150. 
ANTUNES, H. K. M.; STELLA, F. G.; SANTOS, R. F.; BUENO, O. F.; MELLO, M. T. de. Depression, anxiety and quality of life scores in seniors after an endurance exercise program. Revista Brasileira de Psiquiatria. v.27, n.4, p. 266- 271, 2005. Disponível em: http://www.scielo.br/scielo.php? script=sci_arttext\&pid=S151644462005000400003\&Ing=en\&nrm=iso\&tlng=en . doi 10.1590/S1516-44462005000400003.

ARAÚJO, S. R. C. de; MELLO, M. T. de; LEITE, J. R. Transtornos de ansiedade e exercício físico. Revista Brasileira de Psiquiatria.v.29, n.2, p.164- 171, 2007. Disponível em: http://www.scielo.br/pdf/rbp/v29n2/a15v29n2.pdf.

ARAÚJO, K. C. de M.; DEUS, L. A. de; RODRIGUES, F. B.; LOPES, D. de P. L. e O.; BEZERRA, M. E. et. al. Exercício resistido melhora a saúde mental de mulheres de meia-idade. Journal of Physical Education. v.28, n.1, p.1-7, 2017. Disponível em: http://periodicos.uem.br/ojs/index.php/RevEducFis/article/view/31435/19002. doi: 10.4025/jphyseduc.v28i1.2820.

BERTOLUCCI, P.H.F.; BRUCKI, S. M. D.; CAMPACCI, S. R.; JULIANO, Y. O miniexame do estado mental em uma população geral: impacto da escolaridade. Arquivos de Neuropsiquiatria. v. 52, n. 1, p.1-7, 1994.

BIAGGIO, A.; NATALICIO, L.F.; SPIELBERGER, C.D. Desenvolvimento da Forma Experimental em Português do IDATE. Arq Bras Psicol Apl. v.29, p.33-44, 1979. Disponível em:http://bibliotecadigital.fgv.br/ojs/index.php/abpa/article/view/17827/16571.

COELHO, F.H.N.; NATALLI, B.V.A.; BORRAGINE, S.O.F. Benefícios da musculação na terceira idade. EFDeportes.com. v.15, N.148, 2010. Disponível em:http://www.efdeportes.com/efd148/beneficios-da-musculacao-na-terceiraidade.htm.

GOBBI, S.; VILLAR, R.; ZAGO, A.S. Bases teórico-práticas do condicionamento físico. Rio de Janeiro: Guanabara Koogan; 2005.

BORTOLI, C. G.; PIOVEZAN, M. R.; PIOVEZAN, E. J.; ZONTA, M. B. Equilíbrio, quedas e funcionalidade em idosos com alteração da função cognitiva. Revista Brasileira de Geriatria e Gerontologia. v.18, n.3, p. 587- 597, 2015. Disponível em: http://www.scielo.br/scielo.php?pid=S1809-

98232015000300587\&script=sci_abstract\&tlng=pt. doi: 10.1590/18099823.2015.14057.

BRETAN, O.; PINHEIRO, R. M.; CORRENTE, J. E. Avaliação funcional do equilíbrio e da sensibilidade cutânea plantar de idosos moradores na comunidade. Brazilian Journal of Otorhinolaryngology. v. 76, n.2, p.219-224, 2010. Disponível em: http://www.scielo.br/scielo.php?script=sci_arttext\&pid=S1808-86942010000200012. doi: 10.1590/S1808-86942010000200012.

BRUCKI, S. M. D.; NITRINE, R.; CARAMELLI, P.; BERTOLUCCI, P. H. F.; OKAMOTO, I. H. Sugestões para o uso do mini-exame do estado mental no brasil. 
Arquivos de Neuro- Psiquiatria.v.61, n.3, p.777- 781, 2003. Disponível em: http://www.scielo.br/pdf/anp/v61n3B/17294.pdf.

CHEN, H. M.; TSAI, C. M.; WU, Y. C.; LIN, K. C.; LIN, C. C. Randomised controlled trial on the effectiveness of home-based walking exercise on anxiety, depression and cancer-related symptoms in patients with lung câncer. British Journal of Cancer. v.112, n.3, p. 438- 445, 2015. Disponível em: https://www.ncbi.nlm.nih.gov/pmc/articles/PMC4453645/. doi: 10.1038/bjc.2014.612.

COELHO, B.; SOUZA, L. K. de; BORTOLUZZI, R.; RONCADA, C.; TIGGEMANN, C. L. et al. Comparação da força e capacidade funcional entre idosos praticantes de musculação, hidroginástica e não praticantes de exercícios físicos. Revista Brasileira de Geriatria e Gerontologia. v.17, n.3, p.497-504, 2014. Disponível em: http://www.scielo.br/pdf/rbgg/v17n3/1809-9823-rbgg-17-03-00497.pdf. doi: 10.1590/1809-9823.2014.13046.

DOMICIANO, B. R.; BRAGA, D. K. A. P.; SILVA, P. N. da; VASCONCELOS, T. P. de; MACENA, R. H. M. Escolaridade, idade e perdas cognitivas de idosas residentes em instituições de longa permanência. Revista Neurociências. v.22, n.3, p. 330336, $2014 . \quad$ Disponível em: http://www.revistaneurociencias.com.br/edicoes/2014/2203/Original/971original.pdf. doi: 10.4181/RNC.2014.22.03.971.7p.

FILHO, C. A. A. M.; ALVES, C. R. R.; SEPÚlVEDA, C. A.; COSTA, A. dos S.; JUNIOR, A. H. L. et al. Influência do exercício físico na cognição: uma atualização sobre mecanismos fisiológicos. Revista Brasileira de Atividade Física e Saúde. v.20, n.3, p.237- 241, 2014. Disponível em: http://www.scielo.br/pdf/rbme/v20n3/1517-8692-rbme-20-03-00237.pdf. doi: 10.1590/1517-86922014200301930.

GALLI, R ; MORIGUCHI, E.H.; BRUSCATO, N.M; HORTA, R.L. Active aging is associated with low prevalence of depressive symptoms among brazilian older adults. Revista Brasileira de Epidemiologia. v.19, n.02, p. 307-316, 2016. Disponível em:https://scielosp.org/scielo.php?script=sci_arttext\&pid=s1415790x2016000200307. doi: 10.1590/1980-5497201600020008.

HELRIGLE, C.; FERRI, L. P.; NETTA, C. P. de O.; BELEM, J. B.; MALYSZ, T. Efeitos de diferentes modalidades de treinamento físico e do hábito de caminhar sobre o equilíbrio funcional de idosos. Revista Fisioterapia em Movimento. v.26, n.2, p.321-327, 2013. Disponível em: http://www.scielo.br/pdf/fm/v26n2/09.pdf.

LATTARI, E.; PAES, F.; MACHADO, A.; ROCHA, N. B. F.; NARDI, A. E. et al. Efeitos crônicos do exercício aeróbio sobre o transtorno do pânico: uma revisão sistemática de ensaios clínicos randomizados e não-randomizados. Medical Express. v.2, n.6, p.1- 7, 2015. Disponível em: http://www.scielo.br/pdf/medical/v2n6/2318-8111medical-02-06-20150602.pdf. doi: 10.5935/MedicalExpress.2015.06.02.

MACHADO, M. B.; IGNÁCIO, Z. M.; JORNADA, L. K.; RÉUS, G. Z.; ABELAIRA, H. $M$. et al. Prevalência de transtornos ansiosos e algumas comorbidades em idosos: um estudo de base populacional. Jornal brasileiro de psiquiatria. v.65, n.1, p. 28- 
35, 2016. Disponível em: http://www.scielo.br/scielo.php?pid=S004720852016000100028\&script=sci_abstract\&tlng=pt. doi: 10.1590/00472085000000100.

MONTORO, M. V. P.; MONTILLA, J. A. P.; AGUILERA, E. L.; CHECA, M. A. Intervención en la sarcopenia con entrenamiento de resistencia progresiva y suplementos nutricionales proteicos. Revista Nutrición Hospitalaria. v. 31, n. 4, p. 1481- 1490, 2015. Disponível em: http://web.b.ebscohost.com/ehost/pdfviewer/pdfviewer?vid=1\&sid=210b4a53-1cf344c1-9e3b-f10962f070ce\%40sessionmgr104. doi:I0.3305/nh.2015.31.4.8489.

OLIVEIRA, D. V.; BERTOLINI, S. M. M. G.; MARTINS, J. J. Qualidade de vida de idosas praticantes de diferentes modalidades de exercício físico. ConScientiae Saúde. v.13, n.2, p.187-195, 2014. Disponível em: http://web.a.ebscohost.com/ehost/pdfviewer/pdfviewer?vid=1\&sid=5c0e367d-78d4458f-b836-fb1c1f398d13\%40sessionmgr4007. doi: 10.5585/ConsSaude.v13n2.4697.

PADUBIDRI, A.; SNIH, S. A.; SAMPER- TERNENT, R.; MARKIDES, K. S.; OTTENBACHER, K. J. et al. Falls and cognitive decline in Mexican Americans 75 years and older. Clinical Interventions in Aging. v.9, n.7, p.19-726, 2014. Disponível em: https://www.ncbi.nlm.nih.gov/pmc/articles/PMC4003146/.

PENNINX, B. W.; REJESKI, W. J.; PANDYA, J.; MILLER, M. E.; DI BARI, M. et al. Exercise and depressive symptoms: a comparison of aerobic and resistance exercise effects on emotional and physical function in older persons with high and low depressive symptomatology. The Journals of Gerontology, Series B: Psychological Sciences and Social Sciences. v.57, n. 2, p.124-132, 2002. Disponível em: https://www.ncbi.nlm.nih.gov/pubmed/11867660.

QUEIROZ, A. C. C.; BRITO, L. C. de; SANTOS, M. A. dos; FECCHIO, R. Y.; STOCCO, A. L. B. et. al. Prescrição de caminhada não supervisionada, risco cardiovascular e aptidão física. Revista Brasileira de Educação Física e Esporte. v.27, n. 3, p.377- 386, 2013. Disponível em: https://www.revistas.usp.br/rbefe/article/viewFile/63109/65900.

RAMOS, L. H. N.; CHAGAS, E. F. B. Associação entre atividade física habitual e cognição global em mulheres pós-menopausa usuárias do SUS. Revista Ciência e Movimento. v. 23, n. 2, p.14- 21, 2015. Disponível em: https://www.researchgate.net/publication/283174789_Associacao_entre_Atividade_F isica_Habitual_e_Cognicao_Global_em_Mulheres_Pos-

Menopausa_Usuarias_do_SUS. Doi: 10.18511/0103-1716/rbcm.v23n2p14-21.

SPIELBERGER, C.D.; GORSUCH, R.L.; LUSHENE, R.E. Manual for the state-trait anxiety inventory ("self-evaluatin questionaire"). California: Consulting Psychologists; 1970. Disponível em: https://ubir.buffalo.edu/xmlui/handle/10477/2895.

TREWARTHA, K. M.; GARCIA, A.; WOLPERT, D. M.; FLANAGAN, J. R. Fast butfleeting: adaptive motor learning processes associated with aging and cognitive decline. Journal of Neuroscience. v.34, n. 40, p.13411-13421, 2014. Disponível 
em:

https://www.ncbi.nlm.nih.gov/pmc/articles/PMC4180475/.

doi:

10.1523/JNEUROSCI.1489-14.2014.

VARGAS, L. S. de; LARA, M. V. S. de; MELLO- CARPES, P. B. Influência da diabetes e a prática de exercício físico e atividades cognitivas e recreativas sobre a função cognitiva e emotividade em grupos de terceira idade. Revista Brasileira de Geriatria e Gerontologia. v.17, n. 4, p.867-878, 2014. Disponível em: http://www.scielo.br/pdf/rbgg/v17n4/1809-9823-rbgg-17-04-00867.pdf. doi: 10.1590/1809-9823.2014.13178.

World Health Organization. Global recommendations on physical activity for health. Genebra: WHO; 2010. Disponível em:

http://whqlibdoc.who.int/publications/2010/9789241599979_eng.pdf . Acessado em 22 de abril de 2018. 